\title{
Pressure-sensitivity Effects on Toughness Measurements of Compact Tension Specimens for Strain-hardening Solids
}

\author{
Abdulhamid A. Al-Abduljabbar \\ Department of Mechanical Technology, \\ Riyadh College of Technology, \\ Riyadh, Saudi Arabia
}

\begin{abstract}
The path-independent $J$-integral is used as a fracture prediction criterion for loading beyond the elastic limit as Linear Elastic Fracture Mechanics (LEFM) cannot be applied. While $J$ was originally defined from an energy perspective, it was demonstrated that it could be inferred from load-displacement diagrams. Therefore, the Compact Tension (CT) specimen testing is used to compute $J$ for materials. In this work, the $\eta$-factor, an important parameter in the computation of the $J$-integral, is investigated for compact tension specimen for materials with pressure sensitive yielding. This is achieved by using a lower bound approach to derive the appropriate expression from $\eta$ from the test geometry and material properties. The specimen is considered at fully plastic loading where it is in the state of collapse. The effect of pressure sensitivity is accounted for by using a Drucker-Prager yield criterion for solid materials. Since CT testing is usually conducted on metallic materials, strain-hardening behavior of the material is incorporated in this analysis. This is done by assuming a simple linear hardening curve of the material. Numerical results computed for different cases show that as the material strain hardening increases.
\end{abstract}

Key words: $J$-Integral, plastic yielding, toughness, compact tension testing, pressure-sensitive yielding

\section{INTRODUCTION}

The Linear Elastic Fracture Mechanics (LEFM) theory is useful for estimation of fracture behavior of materials in the elastic range. However, as large inelastic strain behavior is encountered, the LEFM is not adequate to describe the toughness and fracture behavior. Attempts to model such behavior is going as early as Rice ${ }^{[1,2]}$ who introduced a path-independent line integral around the tip of the fracture notch to describe the elastic-plastic fracture behavior. Later on, Begley and Landes ${ }^{[3,4]}$ demonstrated that it is possible to estimate the $J$ integral from the load-displacement curves of various test specimen geometries. Bucci et $a l .{ }^{[5]}$ and Rice et $a l^{[6]}$ proposed possible estimation methods of $J$ for compact tension specimens among other specimen geometries.

Later on, Merkle and Corten ${ }^{[7]}$ improved the estimation of $J$ by considering the effects of the bending moment resulting from the applied load to the remaining ligament of the compact tension specimen.

They used a lower-bound approach to derive the $\eta$ factor for the $J$ integral estimation. The $\eta$ factor approach has been used to estimate the $J$ integral from the area under the load-displacement $(P-\Delta)$ curve of a test specimen or structure.

The Von-Mises yield criterion assumes an ideal material yield that only depends on the equivalent yield stress. While such assumption is acceptable for many classes of materials especially metals, it is not the case for other classes of materials such as polymers, phasetransformation ceramics, cast irons and even some classes of steels. In such materials, yielding behavior is affected by the state of hydrostatic stress and the yield envelope on the equivalent stress versus the hydrostatic stress plane $\left(\sigma_{e}-\sigma_{m}\right)$ is not horizontal, but rather having a negative slope. Such phenomenon, which is also known as pressure-sensitivity, is also demonstrated by the variety between the values of yield stress in tension and compression.

The effect of pressure sensitivity on yield can be accounted for using the Drucker-Prager yield criterion, where, a generalized equivalent yield stress is expressed as a function of the effective yield stress and the hydrostatic stress using a pressure sensitivity factor. The pressure sensitivity factor is a material mechanical property dependent on the difference between yield stresses in tension and compression.

The reported values of pressure sensitivity show a wide range of different engineering materials. For example, for polymers it was reported by Sternstein and Ongchin ${ }^{[8]}$, Spitzig and Richmond ${ }^{[9]}$, and Kinloch and Young ${ }^{[10]}$, that the pressure sensitivity factor, $\mu$, has a range of $0.1 \sim 0.25$. In cast irons it has a value around 0.22 , according to Dong et $a l .{ }^{[11]}$. It is also present, to a lesser degree, in some steels as shown by Richmond and Spitzig ${ }^{[12]}$ with $\mu$ has quite a low value 
of 0.064. Phase transformation in some ceramic materials is highly dependent on pressure sensitivity as reported by experimental investigations of $\mathrm{Yu}$ and Shetty ${ }^{[13]}$ and Chen ${ }^{[14]}$ where $\mu$ has a large value up to 0.93 for zirconia-containing phase-transformation ceramics.

Al-Abduljabbar and $\operatorname{Pan}^{[15]}$ incorporated the material pressure-sensitivity, into the calculation of $\eta$ to produce a better estimate of the toughness property, using the same approach and assumption of rigid perfectly-plastic material behavior used in previous works.

Since most metals exhibit strain hardening in various degrees, incorporating the effect of strain hardening on the estimate of fracture toughness as described by $J$ will indeed result in an improvement of this measure of material fracture behavior. AlAbduljabbar ${ }^{[16]}$ considered strain hardening effect on $J$ for normal solids without pressure sensitivity.

In this work, a procedure similar to those adopted in previous works in order to produce expressions for the $\eta$ factor of the $J$ integral for a material with pressure sensitivity and strain hardening. The pressure sensitivity is modeled using a Drucker-Prager yield criterion for solid materials, while strain hardening is depicted by assuming a simple linear hardening model for the material.

\section{PLASTIC LIMIT ANALYSIS}

The Drucker-Prager yield criterion ${ }^{[17,18]}$ is a generalization of the Coulomb rule is solid mechanics where the shear stress required for simple slip is linearly dependent upon the normal pressure on the slip surface. In the D-P criterion, a generalized yield function is proposed as a combination of the effective yield stress $\left(\sigma_{e}\right)$ and the mean stress $\left(\sigma_{m}\right)$ by using the pressure sensitivity factor $(\mu)$ as follows:

$\mathrm{f}(\tilde{\sigma})=\sigma_{\mathrm{e}}+\sqrt{3} \mu \sigma_{\mathrm{m}}=\sigma_{0}$

In equation (1), $\sigma_{e=}\left(S_{i j} S_{i j}\right)^{1 / 2}$, where $S_{i j}$ is the deviatoric stress components defined by $S_{i j}=\sigma_{i j}-\sigma_{m} \delta_{i j}$. Here, $\sigma_{i j}$ represent stress components and $\delta_{i j}$ is the Kronecker delta. The summation convention is adopted for repeated indices. The mean stress $\left(\sigma_{m}\right)$ is defined by the relation $\sigma_{m}=\sigma_{k k} / 3$. The stress quantity $\sigma_{0}$ is the generalized effective tensile stress at the onset of yield.

Considering the uniaxial tensile and compressive loading conditions and applying the yield criterion in equation (1) values for the effective tensile stress $\left(\sigma_{t}\right)$ and the effective compressive stress $\left(\sigma_{c}\right)$ can be obtained as follows:

$$
\sigma_{\mathrm{t}}=\frac{1}{1+\mu^{\prime}} \sigma_{0}
$$

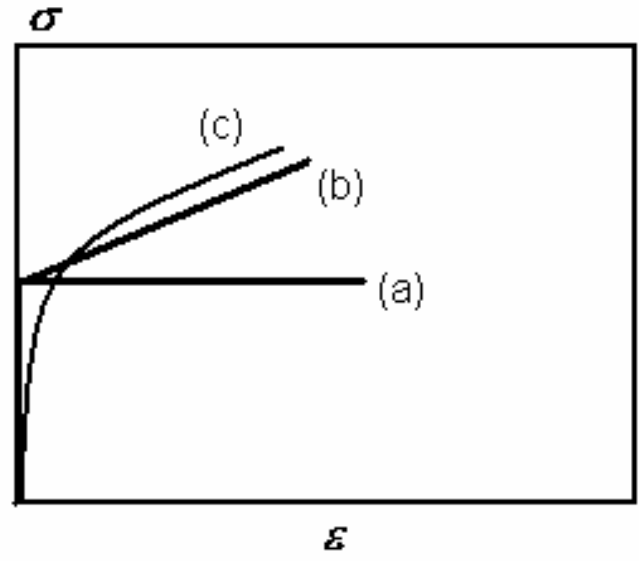

Fig. 1: Stress-strain for different cases: (a) an idealized perfectly-plastic material, (b) an idealized linearly hardening material and (c) a typical ductile metal

$\sigma_{\mathrm{c}}=\frac{1}{1-\mu^{\prime}} \sigma_{0}$

where, $\mu^{\prime}=\mu / \sqrt{3}$.

To describe the material stress strain relation, we adopt the Ludwik's expression ${ }^{[19]}$ :

$\sigma=\sigma_{0}+H \varepsilon^{\mathrm{n}}$

where, $\sigma_{0}$ is the initial yield stress, $H$ is the hardening constant, $\mathcal{E}$ is the strain and $n$ is the hardening exponent. The expression is further simplified by assuming linear hardening case with $n=1$. This relation can be used to describe cold worked metals such as high carbon and alloy steels where there is a high amount of prestraining $^{[19,20]}$.

Shown in Fig. 1 are three curves describing stressstrain behavior. The first one (a) is in an idealized rigid perfectly-plastic material, where the material exhibits no elastic strain and the stress remains at the constant yield value of $\sigma_{0}$ throughout the deformation and plastic straining. Such behavior is the one assumed in previous works for estimation of the $J$ integral ${ }^{[15]}$. The second curve is for a rigid linear hardening material as described by equation (4) with $n=1$.

The third curve is for a typical ductile material. For metals, the elastic strain is usually very small when compared with the plastic strain, especially at high loading levels, as is considered here. This fact permits the assumption of neglecting the contribution of the elastic strain in this analysis.

Next, we consider the compact tension (CT) specimen of a rigid strain-hardening material as shown in Fig. $2^{[21]}$. Due to the symmetry of the specimen geometry and loading condition along the horizontal 
axis, only the upper half of the specimen is analyzed here. The total width of the specimen is $W$, the crack length is $a$, the remaining ligament width is $b$ and the thickness of the specimen (through the paper) is unity. The fully plastic load value applied as shown is $P_{0}$. Also shown in the figure, is the stress distribution in the remaining ligament, where the portion near the tip is under tensile stress resulting from the tension and bending moment of the load and the other portion is under compressive loading. At the point of stress reversal, both compressive and tensile portions start with initial yield stresses with stress levels increasing linearly up to their maximum values at the edges. On the compressive side, the starting stress level is $\sigma_{c}$ and the stress at the outer edge of the specimen is $\sigma_{A}$ and on the tensile side, the starting stress level is $\sigma_{t}$ and the stress at the inner edge is $\sigma_{B}$.

The dimensionless parameter, $\alpha$, is introduced as an indicator of the deviation of the neutral axis from the center of the remaining ligament width $b$, where the tensile side length is $(1+\alpha) c$, because it has lower stress levels and the compressive side length is $(1-\alpha) c$. Within the factor $\alpha$ lies the effect of the pressure sensitivity of the material resulting from the variation of yield stresses. Another dimensionless parameter, $\gamma$, is introduced to account for the hardening effect in the material. Assuming that the slope of the linear hardening curve on the stress diagram is proportional to $\gamma$, the values of the two end stresses are determined as:

$\sigma_{\mathrm{A}}=[1+\gamma] \sigma_{\mathrm{c}}$

$\sigma_{\mathrm{B}}=[1+\gamma] \sigma_{\mathrm{t}}$

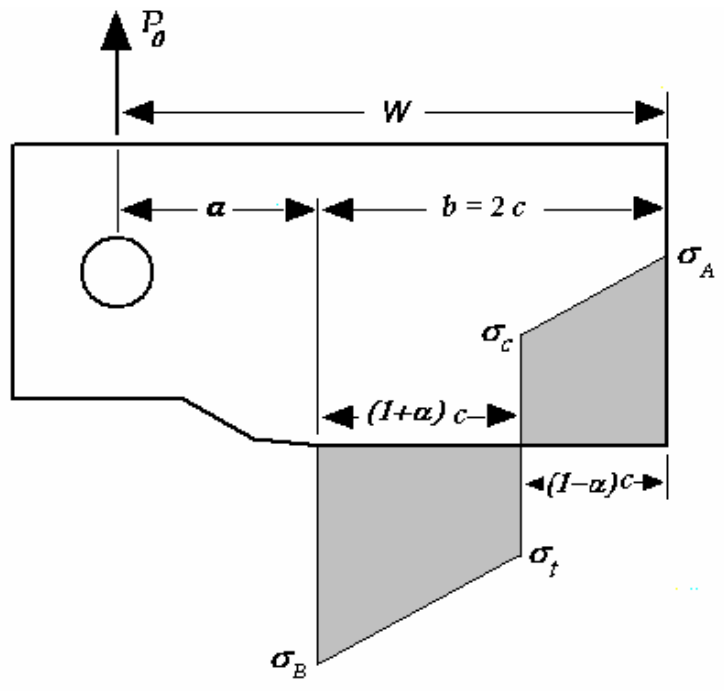

Fig. 2:Geometry of the upper half of the compact tension specimen and the stress diagram for the remaining ligament. Unit thickness is assumed
For the plastic limit load, we consider the axial force and the bending moment equilibrium due to the in-plane force and stresses. The force equilibrium in the vertical direction requires that:

$\mathrm{P}_{0}+\mathrm{c}(1-\alpha) \frac{\sigma_{\mathrm{A}}+\sigma_{\mathrm{c}}}{2}-\mathrm{c}(1+\alpha) \frac{\sigma_{\mathrm{B}}+\sigma_{\mathrm{t}}}{2}=0$

Hence, equation (7) is used with equations (5) and (6) to get the plastic limit load $P_{0}$ as follows:

$\mathrm{P}_{0}=\frac{(2+\lambda)\left(\alpha-\mu^{\prime}\right)}{1-\mu^{\prime 2}} c \sigma_{0}$

Further, the moment equilibrium around stress reversal point requires that the internal resisting moment balances the moment generated by the applied load $M_{p}$. The internal resisting moment is composed of two portions: $M_{t}$ due to the tensile stress and $M_{c}$ due to the compressive stress. This produces:

$\mathrm{M}_{\mathrm{t}}+\mathrm{M}_{\mathrm{c}}-\mathrm{M}_{\mathrm{P}}=0$

The values of these moment components are determined, with the help of equations (2-3, 5-6), and Fig. 2, as follows:

$M_{t}=\left(\frac{1}{2}+\frac{\gamma}{3}\right) \frac{(1+\alpha)^{2}}{1+\mu^{\prime}} c^{2} \sigma_{0}$

$\mathrm{M}_{\mathrm{c}}=\left(\frac{1}{2}+\frac{\gamma}{3}\right) \frac{(1-\alpha)^{2}}{1-\mu^{\prime}} \mathrm{c}^{2} \sigma_{0}$

$\mathrm{M}_{\mathrm{P}}=\mathrm{P}_{0}[\alpha+(1+\alpha) \mathrm{c}]$

Substituting values for moments from equations (10-12) into equation (9) and also using equation (8) for the limit load; a quadratic expression for the parameter $\alpha$ is obtained in the form

$\mathrm{A} \alpha^{2}+\mathrm{B} \alpha+\mathrm{C}=0$

Where:

$$
\begin{aligned}
& \mathrm{A}=(\lambda-1), \\
& \mathrm{B}=\left(\frac{\mathrm{a}}{\mathrm{c}}+1-\mu^{\prime}\right) \lambda+2 \mu^{\prime}
\end{aligned}
$$

And:

$\mathrm{C}=-\left(\frac{\mathrm{a}}{\mathrm{c}}+1\right) \lambda \mu^{\prime}-1$ 
In equations (14-16), $\lambda$ is a dimensionless parameter which is defined by:

$$
\lambda=3\left(\frac{2+\gamma}{3+2 \gamma}\right)
$$

From the above relations, $\alpha$ can be solved for easily using the standard quadratic equation solution. Recalling the work for pressure sensitive rigid perfectly-plastic materials, presented by AlAbduljabbar and $\operatorname{Pan}^{[15]}$, we can see that once we set the strain hardening coefficient to zero, the constants $A, B$ and $C$ of equation (13) above revert to the result given in equation no. 14 in that work, thereby confirming the validity of relations developed here.

\section{THE $\boldsymbol{J}$ INTEGRAL ANALYSIS}

The expression for the $J$ integral for materials with negligible elastic strain can be adopted from the work of Merkle and Corten ${ }^{[7]}$ as follows:

$$
\mathrm{J}_{\mathrm{p}}=\frac{\eta}{\mathrm{b}} \int_{0}^{\Delta_{\mathrm{p}}} \mathrm{Pd} \Delta_{\mathrm{p}}+\frac{\eta^{*}}{\mathrm{~b}} \int_{0}^{\mathrm{P}} \Delta_{\mathrm{p}} \mathrm{dP}
$$

where, $\eta$ and $\eta^{*}$ are parameters dependent on the specimen geometry and loading conditions. The first and second integrals of the equation (18) are the plastic work and the complementary plastic work done on the specimen, respectively. The $\eta$-factor is defined by:

$$
\eta=b \frac{1}{P_{0}} \frac{\mathrm{dP}_{0}}{\mathrm{db}}
$$

As will be shown later, $\eta$ is dependent on the geometry, loading conditions and material behavior. The $\eta$-factor can be determined by first differentiating equation (8) with respect to $b$, noting that $d c / d b=1 / 2$. Then, using equations (8) and (19):

$$
\eta=1+\frac{c}{\alpha-\mu^{\prime}} \frac{\partial \alpha}{\partial c}
$$

Differentiating equation (13) with respect to $c$ and using the relation $a=W-2 c$; we get an expression for the last term in the equation (20):

$$
\frac{c}{\alpha-\mu^{\prime}} \frac{\partial \alpha}{\partial c}=\frac{\lambda\left(\frac{\mathrm{a}}{\mathrm{c}}+2\right)}{2 \alpha(\lambda-1)+\lambda\left(\frac{\mathrm{a}}{\mathrm{c}}+1\right)-\mu^{\prime}(\lambda-2)}
$$

Equation (13) is again used to obtain an expression for $\lambda(a / c+1)$ : $\lambda\left(\frac{\mathrm{a}}{\mathrm{c}}+1\right)=\frac{1+\mu^{\prime} \alpha(\lambda-2)-\alpha^{2}(\lambda-1)}{\alpha-\mu^{\prime}}$

Finally, from equations (20-22), we can determine $\eta$ :

$$
\eta=\frac{2+\mu^{\prime 2}(\lambda-2)-\mu^{\prime} \lambda+\left(1-\mu^{\prime}\right) \lambda \alpha}{1+\mu^{\prime 2}(\lambda-2)-(\lambda-1)\left(2 \mu^{\prime} \alpha-\alpha^{2}\right)}
$$

If the material hardening is removed by setting the parameter $\gamma$ to zero, so that $\lambda=2$, then the equation (23) reduces to

$$
\eta=2 \frac{(1+\alpha)\left(1-\mu^{\prime}\right)}{1-2 \mu^{\prime} \alpha+\alpha^{2}}
$$

This expression for $\eta$ is exactly the same one presented for perfectly plastic material ${ }^{[15]}$. This result for the case without strain hardening serves as a check for the solution obtained from $\eta$ in the analysis above.

\section{DISCUSSION}

The relations that were developed for $\eta$ in the preceding section will be used to assess the effects of the strain hardening and pressure sensitivity on the fracture behavior of metals based on the compact tension specimen parameters. Since those relations are based on the aforementioned assumptions in a previous section, they serve as indicative measures of the material behavior, not direct evaluators of the real property. The relations developed for $\eta$ earlier were presented in terms of $a / c$. It is appropriate to express them here in terms of the normalized crack length $a / W$. This is achieved by recalling from the geometry of the specimen in Fig. 2, that:

$\frac{\mathrm{a}}{\mathrm{c}}=\frac{2 \mathrm{a} / \mathrm{W}}{1-\mathrm{a} / \mathrm{W}}$

We first consider the behavior of the $\eta$ factor as a function of the normalized crack length for a perfectly plastic material and two cases of strain hardening materials $(\gamma=0,0.5$ and 1.0 , respectively) where all materials having no pressure sensitivity $(\mu=0)$. The results for this case are shown in Fig. 3. The figure shows that at low values of crack length the change in the hardness factor produces a change in the value of the $\eta$ factor in the same direction. An increase in the value of $\gamma$ gives an increase in $\eta$, resulting in a higher value of the $J$ integral. The case of no hardening corresponds to the results obtained by previous works $^{[16,17]}$. 


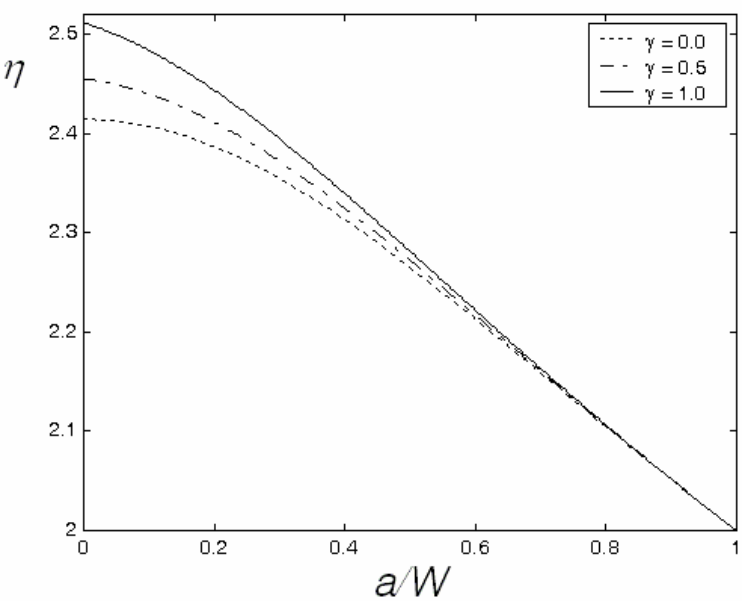

Fig. 3: The $\eta$ factor as a function of the normalized crack length $(a / W)$ for different values of the strain hardening coefficient $\gamma$ and no pressure sensitivity $(\mu=0)$

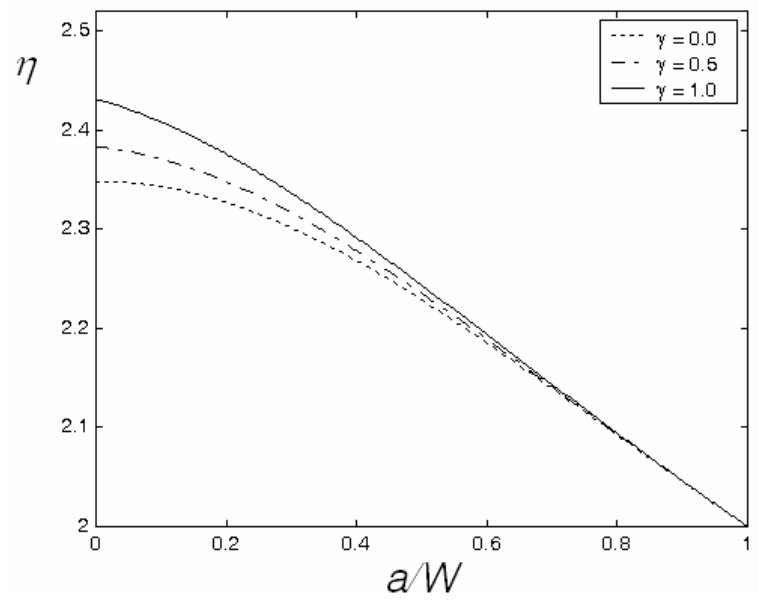

Fig. 4: The $\eta$ factor as a function of the normalized crack length $(a / W)$ for different values of the strain hardening coefficient $\gamma$ and a pressure sensitivity factor $\mu=0.1$

Next, consider the change of $\eta$ with respect to the normalized crack length for different cases of strain hardening coefficient, $\gamma$, for a low value of pressure sensitivity $\mu=0.1$ as depicted in Fig. 4. The same observation that at low values of crack length, the change in the hardness factor produces a change in the value of $\eta$ in the same direction with the increase in $\gamma$ resulting in an increase in $\eta$, although the effect is milder. It can be seen from the figure that the effect of hardening decreases as the crack extension becomes large and converges to the value of 2 because for deeply cracked specimens, the factor approaches 2 regardless of the material constitutive behavior ${ }^{[6]}$.

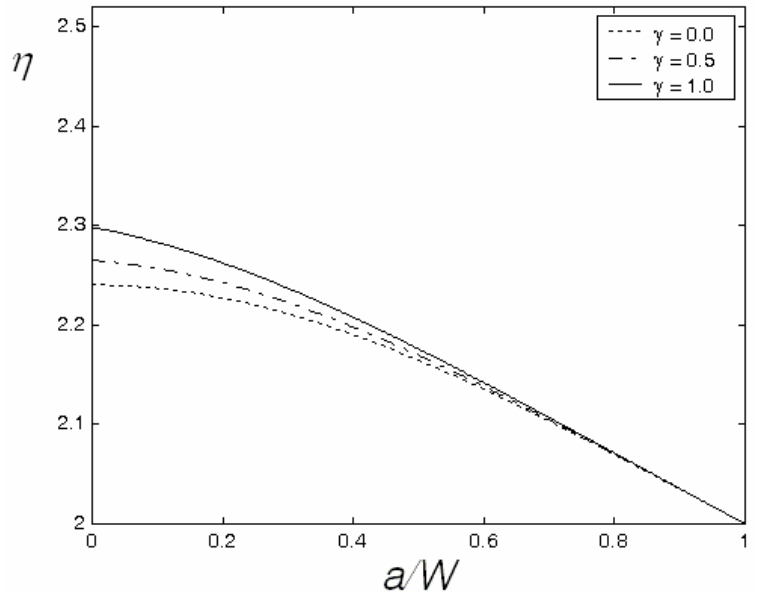

Fig. 5: The $\eta$ factor as a function of the normalized crack length $(a / W)$ for different values of the strain hardening coefficient $\gamma$ and a pressure sensitivity factor $\mu=0.3$

Figure 5 is a plot of $\eta$ as a function of the crack length for a higher value of the pressure sensitivity; $\mu=0.3$. The figure shows the change in $\eta$ as the crack length increases for different cases of strain hardening. By comparison with Fig. 3, we can see that the effect of pressure sensitivity is to reduce the value of $\eta$ while the main features of the dependence of the strain hardening coefficient are the same. It is noted that the change in $\eta$ due to the change in hardening generally gets milder as the strain hardening gets higher and pressure sensitivity tends to reduce the counter balance effects produced by hardening.

\section{CONCLUSION}

In this work, an improved estimate of the factor involved in the evaluation of the integral was obtained. As a fracture toughness criterion for the compact tension specimen, $J$ estimation for strain-hardening pressure-sensitive materials were possible by using plastic limit load analysis and assigning a linear hardening behavior of the specimen material; which provided the basis for derivation of analytical expressions for the relevant parameters.

The numerical results for different cases of pressure sensitivity and strain hardening provide quantification of expected increase in fracture toughness of the material due to hardening when compared with perfectly plastic behavior. The same result is obtained for the decrease due to material pressure sensitivity. The assumptions introduced in the computation of factor limit the validity of the results, requiring a more rigorous model of hardening and also a comparison with experimental data. 


\section{REFERENCES}

1. Rice, J.R., 1968. A path independent integral and the approximate analysis of strain concentration by notches and cracks. J. Appl. Mech., 35: 379-386.

2. Rice, J.R., 1969. Mathematical Analysis in Mechanics of Fracture, in Fracture II. Edited by H. Liebowitz. Academic Press, pp: 191-311.

3. Begley, J.A. and J.D. Landes, 1972. The $J$-integral as a fracture criterion, fracture toughness. Proc. Natl. Symp. Fracture Mechanics, Part II, ASTM STP 514, Am. Soc. Testing and Materials, pp: 120.

4. Landes, J.D. and J.A. Begley, 1972. The effect of specimen geometry on JIC, fracture toughness. Proc. Natl. Symp. Fracture Mechanics, Part II, ASTM STP 514, Am. Soc. Testing and Materials, pp: 24-39.

5. Bucci, R.J., P.C. Paris, J.D. Landes and J.R. Rice, 1972. J-integral estimation procedures, fracture toughness. Proc. Natl. Symp. Fracture Mechanics, Part II, ASTM STP 514, Am. Soc. Testing and Materials, pp: 40-69.

6. Rice, J.R., P.C. Paris and J.G. Merkle, 1973. Some further results of $J$-integral analysis and estimates. Progress in Flaw Growth and Fracture Toughness Testing. ASTM STP 536, Am. Soc. Testing and Materials, pp: 231-245.

7. Merkle, J.G. and H.T. Corten, 1974. A $J$-integral analysis of the compact specimen, considering axial force as well as bending effect. J. Press. Vess. Tech., Trans. ASME, pp: 286-292.

8. Sternstein, S. and L. Ongchin, 1969. Yield criteria for plastic deformation of glassy high polymers in general stress fields. Am. Chem. Soc. Polymers Preprint, 10: 1117-1124.

9. Spitzig, W. and O. Richmond, 1979. Effect of hydrostatic pressure on the deformation behavior of polyethylene and polycarbonate in tension and compression. Poly. Eng. Sci., 19: 1129-1139.
10. Kinloch, A. and R. Young, 1983. Fracture Behavior of Polymers. Elsevier Applied Sciences, London.

11. Dong, M., C. Piour and D. Francois, 1997. Damage influence on the fracture toughness of nodular cast iron: Part II, Damage zone characterization ahead of a crack tip. Metall. Mater. Trans., 28A, 11: 2255-2262.

12. Richmond, O. and W. Spitzig, 1980. Pressure dependence and dilatancy of plastic flow. In Intl. Union of Theor. Appl. Mech., pp: 377-386.

13. Yu, C. and D. Chetty, 1989. Transformation zone shape, size and crack-growth-resistance (R-Curve) behavior of serial-partially-stabilized Zirconia polycrystals. J. Amer. Ceram. Soc., 72: 921-928.

14. Chen, I.W., 1991. Model of transformation toughening in brittle materials. J Amer. Ceram. Soc., 74: 2564-2572.

15. Al-Abduljabbar, A. and J. Pan, 1999. Effects of pressure sensitivity on the $\eta$-factor and the $J$ integral estimation for compact tension specimens. J. Mat. Sci., 34: 1-8.

16. Al-Abduljabbar, A., 2002. Effect of strain hardening on the estimation of $J$-integral in compact tension specimens. King Saud Univ. J., 16, Eng. Sci. 2: 305-317.

17. Drucker, D. and W. Prager, 1952. Soil mechanics and plastic analysis or limit design. Q. Appl. Math., 10: $157-165$.

18. Drucker, D., 1973. Plasticity theory, strengthdifferential (SD) and volume expansion in metals and plastics. Metall. Trans., 4: 667-673.

19. Hill, R., 1983. Mathematical Theory of Plasticity. Oxford University Press, New York.

20. Singh, S., 1990. Theory of Plasticity. Kanna Publishers, Delhi.

21. ASTM, 1989. Standard Test Method for JIC, A Measure of Fracture Toughness. E 813-89 ${ }^{\varepsilon 1}$ Am. Soc. Testing and Materials, PA. 